\section{Monitoring: Informationen abonnieren und strukturieren}

Monitoring, Tracking, Alerting und etliche andere Begriffe beschreiben einen Sachverhalt, der durch das WWW im allgemeinen und durch Web $2.0 \mathrm{im}$ besonderen möglich geworden ist: Sie können Themen, Institutionen, Personen und Events „beobachten“ oder „verfolgen“ und sich die aktuellen Informationen zu den gewünschten Gebieten automatisch zusenden lassen. Dazu zählen nicht nur die Informationen, die von einschlägigen Medien, Institutionen und Personen generiert werden, sondern auch Informationen, die durch Konversationen im Netz entstehen. Es liegt dabei auf der Hand, dass letztere Informationen umfangreicher und disparater sind, aber auch (be-)wertender, was wiederum eine eigene Qualität hat. Deshalb heißt Monitoring nicht nur Sammeln, sondern möglichst auch Filtern, so dass man sich zwar auf dem Laufenden hält, aber möglichst spezifische und geringe Informationsmengen zur Kenntnis nehmen muss.

\subsection{Alerting-Methoden}

\subsubsection{RSS-Feed}

Feeds sind überall, sie müssen sie nur sehen! Viele Seiten bieten das orangefarbene Icon

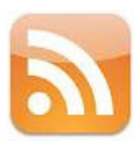

als Visualisierung, damit man es schnell erkennt, bei vielen wird dieses Icon auch oben in der Adresszeile angezeigt, wenn Sie im Browser das entsprechende Add-on geschaltet haben, für den Firefox das Add-on RSS Icon [https://addons.mozilla.org/de/firefox/addon/rss-icon/], für den Chrome die Erweiterung „,RSS-Abonnement".

Was sind Feeds? Sie dokumentieren die Aktualisierung einer Webseite, oft nicht nur die Aktualisierungen von Texten, sondern auch von Multimediadokumenten wie Bildern, Videos oder Audiodateien. Sie bekommen also mit, wenn die Bebilderung einer Museumsseite sich ändert, Sie sehen, wenn neue Videos in ein Blog eingestellt werden, Sie lesen, was in den „Aktuelles“-Meldungen einer Institution steht. Was sich auf den verschiedenen Web 2.0-Diensten tut - in Weblogs, Wikis, Bookmarksammlungen, Fotorepositorien und vielen anderen mehr - diese Dienste haben sowieso RSS-Feeds „,an Bord“, sind also in dieser Hinsicht hervorragend erschlossen.

\subsubsection{Mailing-Alerts}

Wo es keine Feeds gibt, gibt es oft die Option, E-Mail-Alerts einzurichten. Beispielsweise bei Datenbanken oder Homepages von eJournals kann man oft seine E-MailAdresse eingeben und bekommt dann die Aktualisierungen zugesandt.

Hier ist es wichtig, Ihr E-Mail-Programm richtig einzustellen (s. oben unter „Kommunikation"), damit Sie solche Benachrichtigungen nicht als Belästigung empfinden: Setzen Sie Filter, so dass die betreffenden Nachrichten in einem („Alerts“) oder
Lernziele

In diesem Kapitel lernen Sie, mit Hilfe einfacher Techniken Neuigkeiten über Themen, Institutionen und Personen zu verfolgen, so dass Sie automatisch auf dem Laufenden bleiben.

Inhalt dieses Kapitels:

- Alerting-Methoden

- Umwandeln und Bündeln

- RSS-Reader

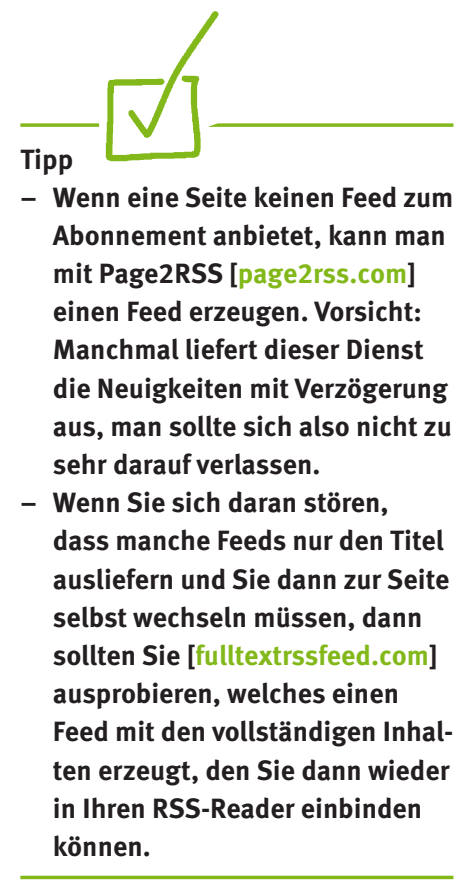



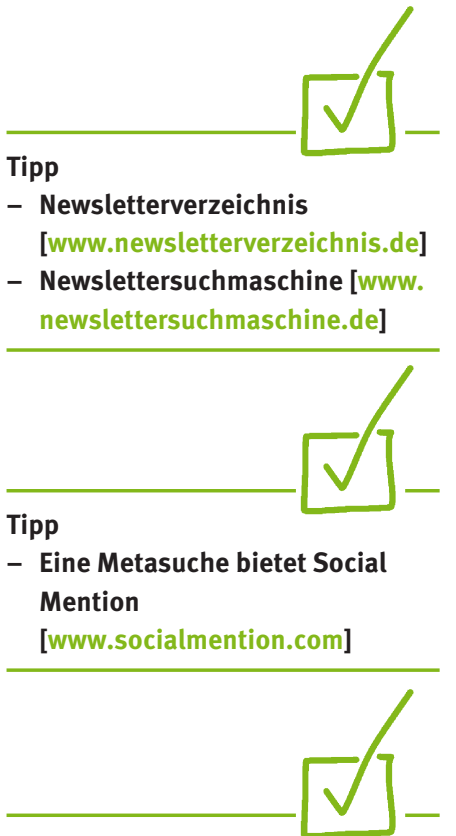

Tipp

- Twitter-Suche [search.twitter.com]

- Topsy [topsy.com] ist eine Spezialsuchmaschine für Twitter, die neben einem RSS-Feed auch die Option eines E-Mail-Alerts bietet

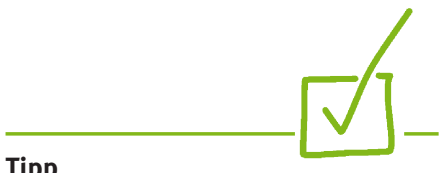

Eine Linksammlung, die so gut wie alle relevanten Tools in diesem Bereich umfasst, ist Feed Software [www.feed-software.de]. mehreren thematisch formulierten Ordnern gesammelt werden und Sie diese dann, wenn Sie Zeit haben, gesammelt durchsehen können!

\subsubsection{Newsletter}

Die hohe Zeit der digitalen, meist per E-Mail versandten Newsletter war Mitte der neunziger Jahre.

Aber auch heute noch versenden Institutionen gern gut formatierte Newsletter an durchaus nicht kleine Verteiler. Sie werden also immer noch gern gelesen! Meist werden sie per E-Mail verschickt, so dass Sie sie wie die E-Mail-Alerts filtern können.

\subsubsection{Twitter}

Soziale Dienste, heute gern „Social Media“ genannt, bieten mittlerweile viele Möglichkeiten, bestimmte Themen zu beobachten, die im Schwange sind.

Wenn man nun einen Dienst nehmen will, der zuverlässig Trendthemen transportiert, dann ist Twitter das Mittel der Wahl, wenn man diese Themenmeldungen so verarbeiten will, dass sie mit anderen Quellen kombinierbar sind. Die Twittersuche bietet die Möglichkeit, einen RSS-Feed zu abonnieren, so dass man Meldungen im Reader zusammen mit anderen Quellen lesen kann.

Bezüglich Twitter sollte noch angemerkt werden, dass es sich hier um eine „Echtzeit“-Quelle handelt. Immer dann, wenn Sie auf aktuellste Meldungen angewiesen sind, ist Twitter das Mittel der Wahl. Dafür sind die Einträge aber auch nach ca. 30 Tagen nicht mehr auf Twitter zu recherchieren, hier müssen Sie auf Suchmaschinen wie Topsy ausweichen! Die hier geschilderten Quellen lassen sich umwandeln und zusammenfassen; so dass man nicht viele verschiedene Einträge durcharbeiten muss.

\subsection{Umwandeln und Bündeln}

\subsubsection{Konverter}

Sie verwandeln ein Format in ein anderes, um die Informationen einheitlich weiter bearbeiten zu können. Beim Konvertieren leisten verschiedene Tools gute Dienste, wie beispielsweise xfruits [www.xfruits.com] oder rss2email [www.allthingsrss.com/ rss2email]. Die einzelnen Meldungen eines RSS-Feeds werden mit ihrer Hilfe in eine E-Mail umgewandelt und an ein vorher bestimmtes Konto gesandt. So hat man dann die Chance, die Informationen auf einer Plattform zusammenzuführen und dort eventuell zu filtern, zur Kenntnis zu nehmen und weiter zu verarbeiten.

\subsubsection{Aggregatoren}

Neben dem Umwandeln in ein anderes Format ist es wichtig, parallele Quellen möglichst zusammenzuführen, so dass man sie in einem Fluss oder parallel zur Kenntnis nehmen kann. Das ist außerordentlich zeitsparend, denn bei Suchdiensten, die zur Erschließung keine Schlagwörter bieten, muss man oft die ganze Bandbreite möglicher Suchbegriffe einschließlich ihrer Übersetzungen anwenden, um alle relevanten 
Informationen einsammeln zu können. Sie können einen RSS-Reader als Aggregator einsetzen, andere Aggregatoren sind im Netz für bestimmte Themen verfügbar, wie z. B. für bibliothekarische Weblogs der Plan3t [www.plan3t.info].

\subsection{RSS-Reader}

Für das Abonnieren und Lesen von Feeds benötigt man RSS-Reader, die auf ganz unterschiedlichen Ebenen zur Verfügung stehen. Entweder können Sie Feeds in Ihrem E-Mail-Programm verwalten - Thunderbird [www.mozilla.org/de/thunderbird] ist dafür ein Beispiel -, Sie können die Feeds auch als „dynamische Bookmarks“ im Firefox- oder IE-Browser zusammen mit den Lesezeichen verwalten, haben hier aber lediglich die Überschriften der Einträge und müssen für den Inhalt jeweils auf die Originalquelle wechseln oder Sie installieren einen Feedreader als Programm auf Ihrer Festplatte. Unbedingt zu empfehlen sind webbasierte RSS-Reader, die von verschiedenen Geräten aus gelesen werden können (selbst mit Hilfe von Smartphones!) und darüber hinaus auch gute Darstellungs- und Erschließungsfunktionen bieten.

\subsubsection{Google Reader: Schritt für Schritt}

Der Feedreader Google Reader [www.google.com/reader] bietet die Möglichkeit, die verschiedenen Feeds mithilfe von Schlagwörtern zusammenzufassen und so die Meldungen themenorientiert zur Kenntnis zu nehmen.

Den Google Reader möchten wir Ihnen hier Schritt für Schritt vorstellen. Zum einen ist er derzeit der RSS-Reader mit der besten Konfiguration, zum anderen kann man an seinem Beispiel sehr schön den Leistungsumfang von RSS-Readern insgesamt zeigen. Denn man hört in letzter Zeit immer öfter das Argument, RSS sei nicht mehr relevant, Facebook oder andere soziale Netzwerke würden ausreichen, um sich auf dem Laufenden zu halten. Facebook kann in der Tat solche Funktionalitäten bieten. Nur ist es so, dass der Umfang der Quellen, die man mit Facebook einbinden und strukturieren kann, sehr zu wünschen lässt. Hinzu kommt das Argument der Portabilität Ihrer Sammlung: Sie können im Google Reader und anderen RSS-Readern jederzeit Ihre Sammlung exportieren (die Quellen, freilich nicht die Inhalte der Quellen) und in einer anderen Anwendung wieder importieren. Mit sozialen Netzwerken geht das nicht.

Für den Google Reader benötigen Sie einen Account bei Google. Wenn Sie dagegen Bedenken haben, lesen Sie sich die folgenden Absätze durch und richten einen Account bei der kleinen Firma Netvibes [www.netvibes.com] ein, das man ähnlich wie den Google Reader strukturieren kann.

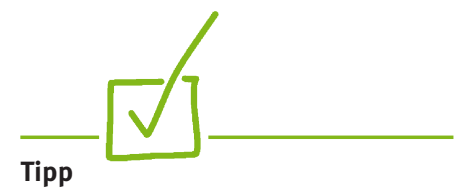

Der Google Reader hat eine Anmutung wie ein E-Mail-Programm. Wenn Ihnen das nicht gefällt, gibt es mit Feedly eine Zusatz-Option. Feedly [www.feedly.com] wird zunächst auf der Basis eines Google Reader-Accounts erstellt, kann dann aber mithilfe eines Add-ons in den Browsern Firefox oder Chrome ergänzt werden. Das Layout von Feedly ist in der Standardansicht zeitungsähnlich, kann aber auch mit mehreren Optionen in anderer Struktur dargestellt werden.

Tipp

Wenn Sie eine größere Sammlung von Feeds haben, zu der immer wieder etwas hinzukommt, dann sollten Sie von Zeit zu Zeit Ihre Sammlung exportieren. Backups zahlen sich aus! 
Sie gehen auf [www.google.com/reader] und loggen sich ein. Dann bekommen Sie eine Ansicht, die so aussehen dürfte:

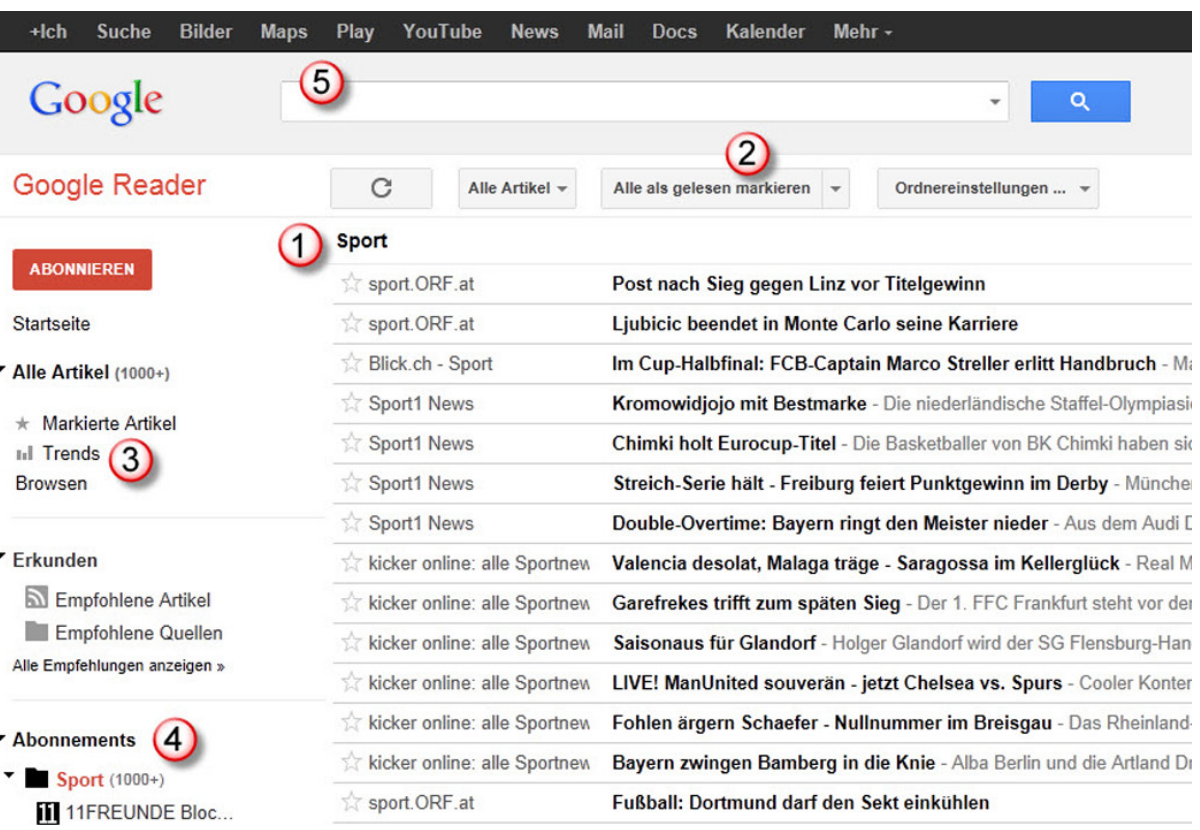

Sie haben rechts einen großen Sektor (1), in welchem die Feeds, also die Nachrichten angezeigt werden. Welche Optionen Ihnen hier zur Verfügung stehen, können Sie mit den Optionen beeinflussen, die in der Leiste über diesem Sektor angezeigt werden (2). Insbesondere kann man hier umschalten zwischen der Kurzanzeige und einer Komplettanzeige der Feeds.

Links finden Sie eine Spalte, in der oben weitere Optionen angezeigt werden (3) und unten die Tags oder Schlagwörter zu sehen sind, die Sie vergeben haben (4). Da Sie noch keine Feeds abonniert haben, wird hier natürlich noch nichts angezeigt. Im Beispiel wurden einige Feeds aus Medien zum Thema Sport abonniert.

Wichtig ist oben das Suchfenster (5), mit dessen Hilfe Sie differenziert in Ihrer Sammlung recherchieren können. Der Mehrwert dieser Funktionalität erschließt sich einem erst, wenn man einmal nach einem Begriff gesucht hat, der schon mehrmals in Beiträgen angezeigt wurde, die man aber auf die Schnelle nicht „heben“ kann. 
Wie fügen Sie nun Feeds in Ihre Sammlung ein? Auf ganz unterschiedlichen Wegen. Entweder, indem Sie auf einer Webseite, auf der ein Feed angeboten wird, auf das RSS-Icon klicken und der Browser (zumindest die drei von uns beschriebenen verfahren so) Sie fragt, wo er denn den Feed einfügen darf. Wählen Sie Google und dann Google Reader aus, dann öffnet sich der Google Reader, und entweder ist der Feed bereits abonniert oder Sie müssen noch einmal bestätigen.

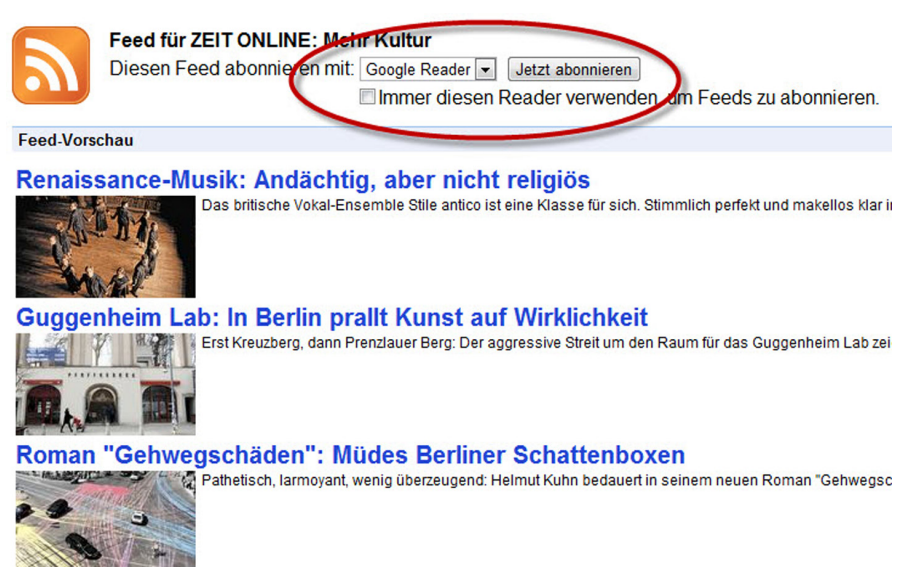

Der zweite Weg wäre, die Adresse (URL) eines Feeds in die Zwischenablage zu nehmen (Rechtsklick auf den blau unterlegten Link und im aufklappenden Menü „Adresse speichern“ auswählen) und dann in den Google Reader einzufügen, indem Sie den roten Button „Abonnieren“ links oben anklicken. Adresse mit Ctrl/Strg+V einfügen (wahlweise auch Rechtsklick und „Einfügen“ anklicken), bestätigen, fertig.

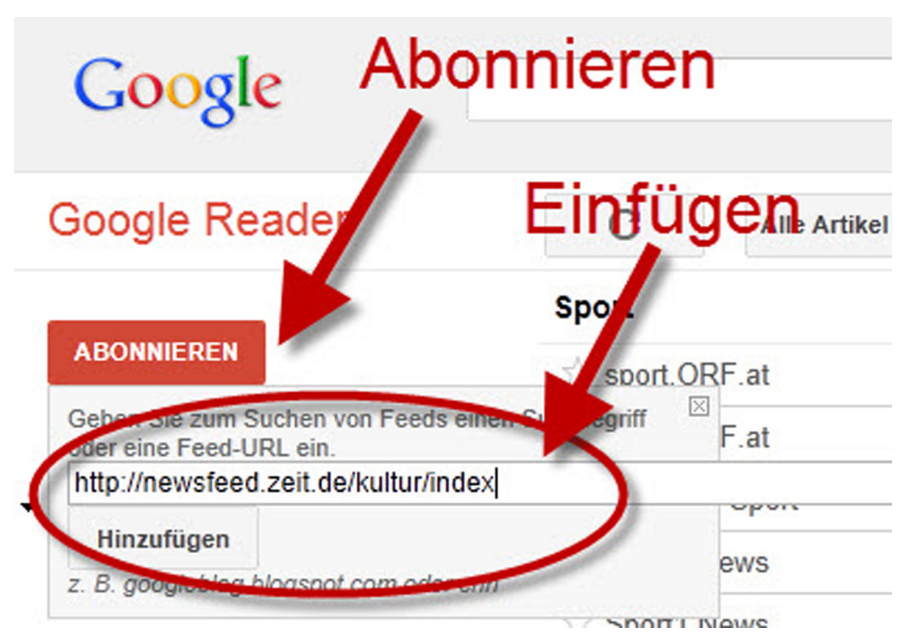


Die dritte Option empfehlen wir dann, wenn Sie keinen konkreten Feed haben, sondern Feeds suchen. Links „Browsen“ anklicken, dann die mittlere Registerkarte „Suchen“ auswählen und Stichwörter zu Ihren Interessen in das Suchfeld eingeben. Sie bekommen dann Feeds vorgeschlagen, die Sie mit einem Klick abonnieren können:

Google Reader Suchergebnisse

\section{ABONNIEREN}

Startseite

- Alle Artikel (1000+)

$\star$ Markierte Artike

Inl Trends

Browsen

Erkunden

\$ Empfohlene Artikel

Empfohlene Quellen

Alle Empfehlungen anzeigen $\gg$

- Abonnements

- Sport $(1000+)$

11 11FREUNDE Bloc..

[20 Minuten | Sport

\section{«Zur Feed-Auswahl zurückkehren}

\section{Abonnenten: Kulturmanagement Net (@kmnweimar) on Twitter}

$1 \quad$ Sign up for Twitter to follow Kulturmanagement Net (@kmnweimar). KM Kulturmanagement Network GmbH. Informationsservice für Fach- und Führungskräfte im .. http://twitter.com/statuses/user_timeline/122677901.rss - Posts pro Woche: 28,5

+ Abonnieren

Abonnenten: Institut für Kulturmanagement Ludwigsburg-Neueste Nachrichten

3

Hier finden Sie Informationen über das Masterstudium "Kulturwissenschaft und Kulturmanagement", das Kontaktstudium Kulturmanagement, über unsere http://www.kulturmanagement. ph-ludwigsburg.de/index.php?id=60\&type=100 - Posts pro Woche: 0,0

† Abonnieren

\section{Abonnenten: Das Kulturmanagement Blog}

$296 \quad$ Kulturmanagement-relevante Themen wie zum Beispiel Projektmanagement und Finanzierung, aber auch Marketing und Kommunikation, http://kulturmanagement.wordpress.com/feed/ - Posts pro Woche: 0,9

$\mp$ Abonnieren 
Übrigens keine Scheu! Das Abwählen von Abonnements geht ebenso schnell wie das Abonnieren. Aber zunächst haben Sie das Problem, dass die neu abonnierten Feeds links unten ungeordnet aufgelistet sind, je mehr es sind, desto unübersichtlicher wird es. Folglich müssen Sie strukturieren und sortieren. Gehen Sie dazu mit dem Mauszeiger auf den Feed (1), rechts dahinter erscheint plötzlich ein kleines schwarzes Dreieck. Da klicken Sie drauf, bekommen ein Pop-up-Menü (2) und können jetzt den Feed entweder bestehenden Schlagwörtern zuordnen (3) oder ein neues Schlagwort vergeben. Übrigens können Sie ohne weiteres mehrfach zuordnen, dann wird der Feed in mehreren Kategorien gelistet.

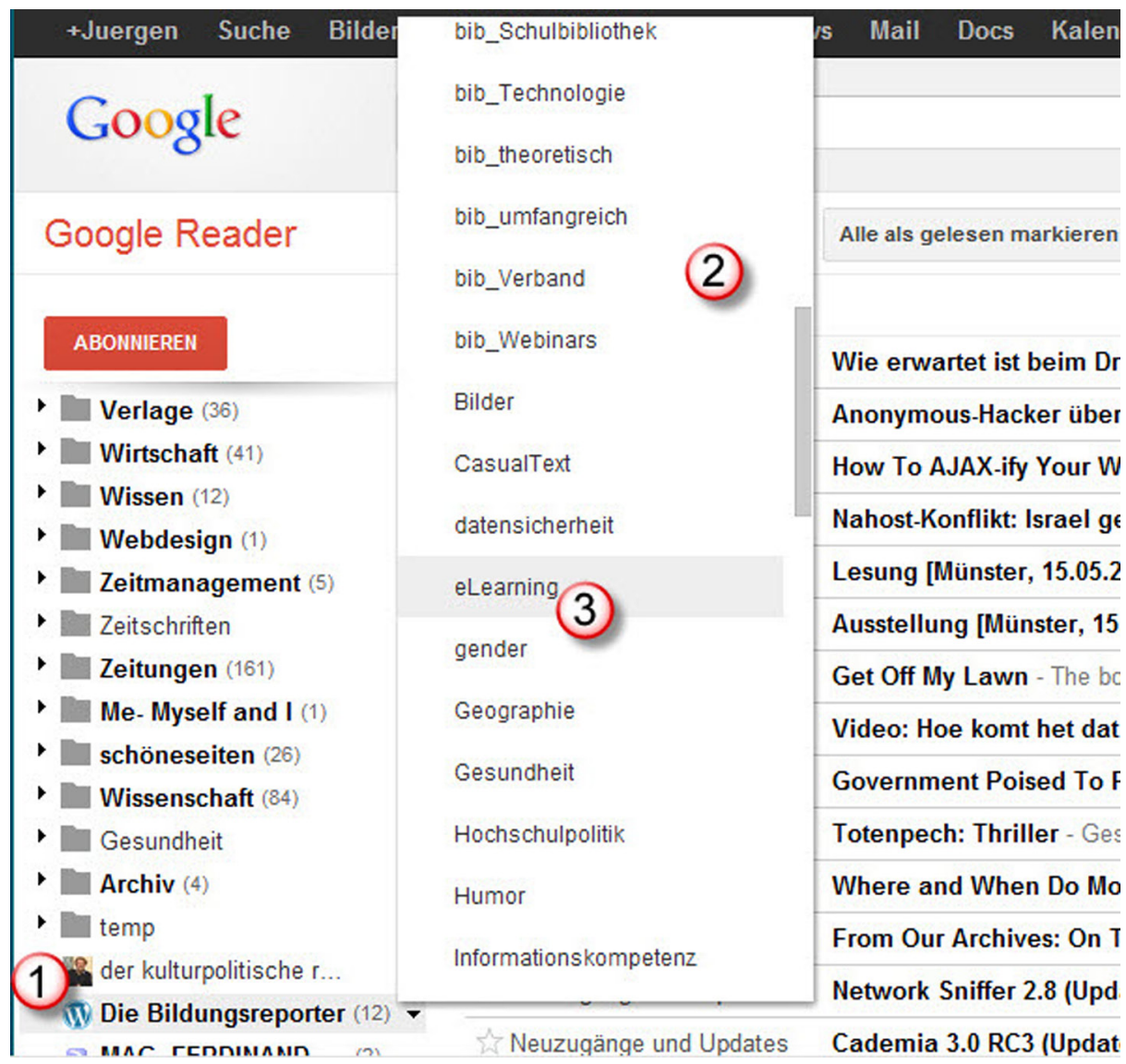

Die Schlagwörter werden dann links über den ungeordneten Feeds angezeigt, lassen sich per drag \& drop verschieben, so dass Sie die wichtigen Kategorien oben haben. Es lohnt sich übrigens, auch formale Kategorien wie „Muss gelesen werden“ oder „Muss nicht gelesen werden“ anzulegen, das spart Zeit. Sicher fragen Sie sich, warum „Muss nicht gelesen werden“ eine Rolle spielt, man könnte die entsprechenden Quellen doch auch abbestellen. Stimmt, nur kommt hier die Suchoption ins Spiel: Wir haben viele Feeds, die wir nicht oder nur selten lesen, in denen aber Relevantes zu neuen Entwicklungen steht. Ist ein bestimmter Begriff neu im Umlauf, kann man mit Hilfe der Suche nachprüfen, ob er bereits in den Quellen verwendet wurde. 


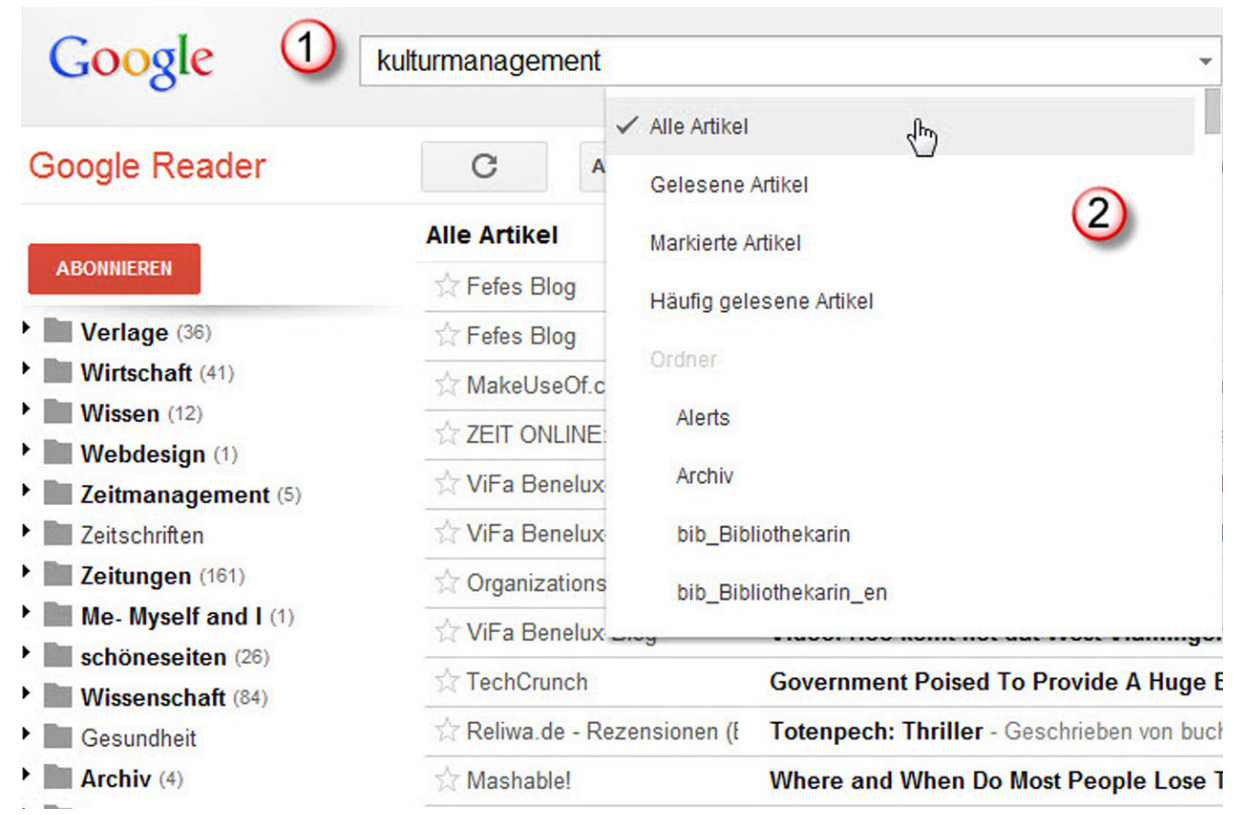

Sie geben in das Suchfeld (1) einen Suchbegriff ein und können dann in einem Aufklappmenü (2) den Suchbereich festlegen - von allen Einträgen bis zu Einträgen einzelner Schlagwörter.

Bleibt zum Schluss noch die Darstellung der einzelnen Einträge der Feeds in der Mitte. Hier können Sie so konfigurieren, dass die Darstellung Ihren Vorlieben und Ihrer Arbeitsform entspricht. Beispielsweise ist es manchmal sinnvoll, nur ungelesene Einträge anzeigen zu lassen, ein andermal ist es richtig, gleich die vollständigen Einträge zeigen zu lassen, beispielsweise bei Bildblogs. Manchmal liest man lieber gebündelt die Einträge zu den einzelnen Schlagwörtern, manchmal ist auch das zu viel und man liest nur die wichtigsten Schlagwörter. Und wenn auch das zu viel ist: Wir haben innerhalb der Schlagwörter die Quellen nochmals nach Wichtigkeit geordnet. Bei hohem Arbeitsaufkommen genügt es dann, die ersten ein bis drei Quellen zu lesen.

\section{Google Reader}

\section{ABONNIEREN}

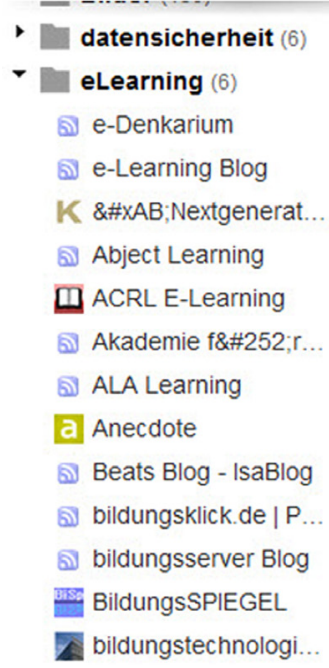


Sie haben noch weitere Möglichkeiten. So können Sie zum Beispiel Einträge mit Sternchen markieren, um sie besser wieder aufzufinden. Und in der Verwaltung - dem Zahnrad-Icon rechts über dem Textfeld - können Sie alles beeinflussen, was die Quellen und die Struktur anbelangt.

Der Reader ist ein Arbeitsinstrument allerersten Ranges, das Ihnen Zeitung, Gerüchteküche, Fortbildung und manches mehr zu ersetzen vermag. Gerade die große Anpassungsfähigkeit der Struktur an die eigenen Interessen ist Gold wert: Sie bestimmen, was Sie in welcher Struktur zur Kenntnis nehmen wollen und Sie bekommen es frei Haus. So ist eine Vielfalt an Quellen, die man sonst mühselig dokumentieren und zeitaufwändig periodisch besuchen müsste, leicht im Blick zu behalten. Setzen Sie den Google Reader - oder einen anderen Feedreader - gezielt ein, um sich in Bezug auf Ihr professionelles und institutionelles Umfeld mit geringem Aufwand auf dem Laufenden zu halten.

\subsubsection{Pocket}

Oft will man etwas nicht jetzt, sondern später lesen und nicht hier, sondern woanders. Hierfür wurde Pocket programmiert, ein Werkzeug, das auf den unterschiedlichsten Geräten installiert werden kann, auf denen jeweils die Sammlung dessen, was man noch lesen will, synchronisiert wird. Gesammelt wird hier mit Hilfe eines Bookmarklets. Egal, womit man auf seinen Pocket-Account zugreift, man hat stets die Sammlung von Dokumenten, Bildern und Videos, die man noch lesen/ansehen wollte, zur Hand, da die Inhalte selbst auf den Server transportiert werden.

Die Integration von Poket mit Sozialer Software ist gut, es besteht eine Interaktion mit vielen Anwendungen. Bemerkenswert ist jene mit Google Reader, in den bei jedem Eintrag ein kleines Plus integriert werden kann, durch welches der Inhalt in Pocket importiert wird.

Pocket bietet auch eine App für IOS und Android an, die das Lesen unterwegs, auch offline, jederzeit möglich macht.

\subsubsection{Instapaper}

Ein weiteres Werkzeug für das Abspeichern von Seiten zum späteren Lesen der Inhalte ist Instapaper [www.instapaper.com]. Um Seiten zu speichern, verwendet auch Instapaper ein Bookmarklet.

Instapaper ist als Desktop-Client, für iPhone und iPad und den Kindle erhältlich. Für die mobilen Endgeräte steht auch das Lesen der Inhalte im Offline-Modus zur Verfügung.

\subsection{Persönliche Startseiten}

\subsubsection{Netvibes}

Netvibes [www.netvibes.com] ist eine so genannte „Startseite“, die in der Anzeige der einzelnen Feeds wie der Google Reader konfiguriert werden kann. Er kann aber auch die verschiedenen Themen und Suchen durch Registerblätter und Fenster (so genannte Widgets) so strukturieren, dass die Darstellung der Feeds äußerst transparent ge-

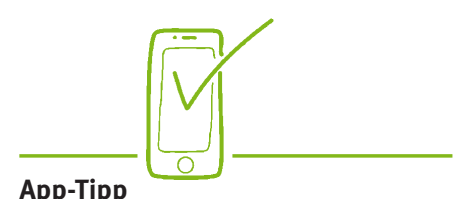

Für IOS-Geräte ist die App Flipboard ein „must have“ für das Lesen von Online-Nachrichten via RSS und aus Sozialen Netzwerken.

Die IOS-App Reeder bietet Ihnen ebenfalls einen sehr angenehmen Lesekomfort für das Lesen von Online-Nachrichten. Beide Apps lassen sich mit dem Google-Reader verbinden.

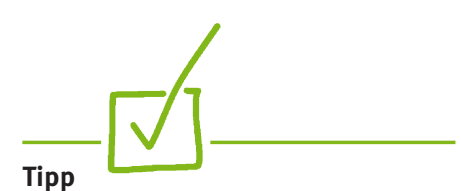
Eine Alternative zu Pocket ist Diigo [www.diigo.com] (vgl. Kapitel 3), das auch eine solche Funktion bietet und Dokumente speichert. Bietet allerdings keinen so großen Komfort bei Smartphones.

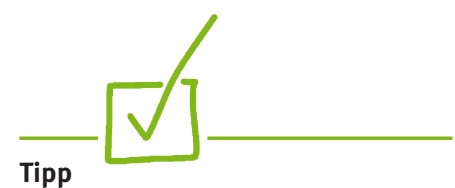
Man muss nicht bei Netvibes registriert sein, um es auszuprobieren! Wenn Sie Netvibes im Browser geladen haben, können Sie einfach ein, zwei Stichwörter eingeben, und Netvibes setzt das in eine typische Struktur mit mehreren Registerblättern und jeweils in diesen etlichen Widgets um. 


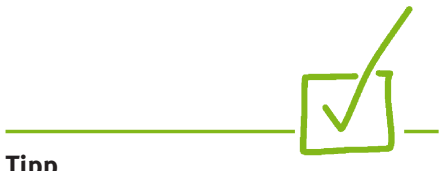

Weitere „Startseiten“ neben netvibes wären zum Beispiel iGoogle [www.google.de/ig] oder MyYahoo! [my.yahoo.com/]

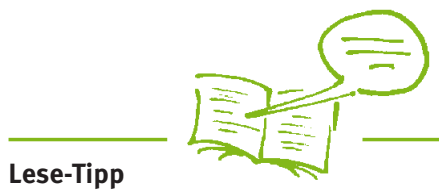

Plieninger, Jürgen: Tools für das Monitoring. - in: Handbuch Internet-Suchmaschinen $2 /$ hrsg. Dirk Lewandowski. - Heidelberg : AKAVerl., 2011. - S. 323-349.

[nbn-resolving.de/ urn:nbn:de:0290-opus-11302] Hier wird auf die verschiedenen Quellen und Werkzeuge für das Monitoring eingegangen. ordnet ist. Die folgende Darstellung bezieht sich auf diese Einstellung, da Netvibes in dieser Konfiguration sehr zeitsparend ist.

Die Darstellung in Registerblättern (1) hilft, die abonnierten Feeds thematisch zu ordnen. Die Widgets (2) können frei verschoben und in ihrer Länge konfiguriert werden, so dass die wichtigsten oben auf der Seite stehen und gleich zur Kenntnis genommen werden können.

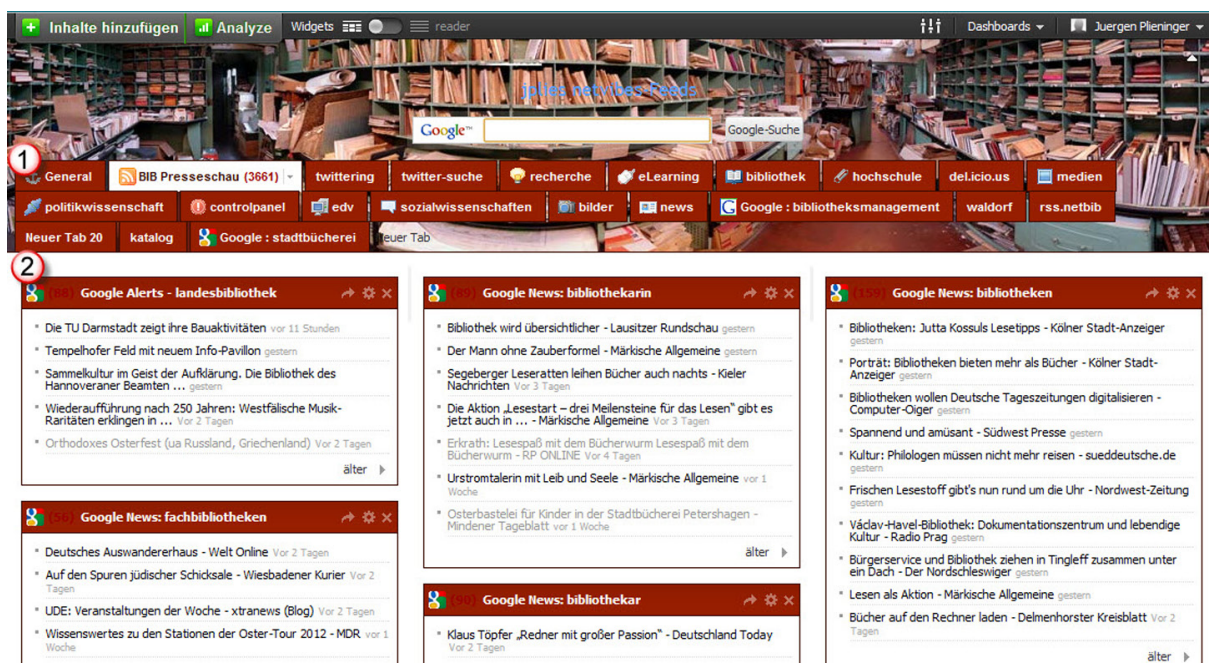

Sie können sich das Registerblatt auch komplett und aktuell auf netvibes ansehen [www.netvibes.com/jplie\#BIB_Presseschau]. Es ist die simultane Abfrage vieler Stichworte bei der Spezialsuchmaschine GoogleNews und bietet so einen aktuellen und schnellen Überblick über Zeitungsartikel zum Thema „Bibliothekswesen in Deutschland“. Netvibes erlaubt eine gut abgestufte Kenntnisnahme der Einträge:

1. Man kann mit dem Blick recht schnell über die Widgets fahren und so die Titel der Einträge „scannen“.

2. Klingt etwas interessant, klickt man es an und dann öffnet sich in Netvibes ein Fenster mit einem „Teaser“, also Kurztext.

3. Erst wenn man dann den ganzen Eintrag lesen möchte, wird man per Link zur Originalseite geleitet.

Eine sehr zeitsparende Abstufung! Bereits gesehene Einträge werden auf der Hauptseite grau gesetzt, so dass man gleich weiß, was man bereits angesehen hat. 